\title{
LMCD1 antisense RNA 1 (LMCD1-AS1) potentiates thyroid cancer cell growth and stemness via a positive feedback loop of LMCD1- AS1/miR-1287-5p/GLI2
}

\author{
Jie Shao ${ }^{1 \#}$, Yonggang $\mathrm{Xu}^{1 "}$, Haixia $\mathrm{Li}^{2}, \mathrm{Lu} \mathrm{Chen}^{3}$, Wenjuan Wang ${ }^{2}$, Danfeng Shen ${ }^{4}$, Jinhong Chen ${ }^{1}$ \\ ${ }^{1}$ Department of General Surgery, Huashan Hospital, Fudan University, Shanghai, China; ${ }^{2}$ Department of Pathology, Huashan Hospital, Fudan \\ University, Shanghai, China; ${ }^{3}$ Department of Pharmacy, Huashan Hospital, Fudan University, Shanghai, China; ${ }^{4}$ Department of Nursing, Huashan \\ Hospital, Fudan University, Shanghai, China \\ Contributions: (I) Conception and design: J Shao, Y Xu, J Chen; (II) Administrative support: J Chen; (III) Provision of study materials or patients: H \\ Li, L Chen, W Wang, D Shen; (IV) Collection and assembly of data: J Shao, Y Xu; (V) Data analysis and interpretation: J Shao, Y Xu, J Chen; (VI) \\ Manuscript writing: All authors; (VII) Final approval of manuscript: All authors. \\ "These authors contributed equally to this work. \\ Correspondence to: Jinhong Chen. Department of General Surgery, Huashan Hospital, Fudan University, No.12 Urumqi Middle Road, Shanghai \\ 200040, China. Email: jinhongch@hotmail.com; Haixia Li, Department of Pathology, Huashan Hospital, Fudan University, No.12 Urumqi Middle \\ Road, Shanghai 200040, China. Email: haixia-li@126.com.
}

Background: LMCD1 antisense RNA 1 (LMCD1-AS1) is a certified oncogene in several tumour types. However, its role in thyroid cancer (THCA) remains unknown.

Methods: The expression level of LMCD1-AS1 in THCA cells and the normal control cell was measured by quantitative real-time polymerase chain reaction (qRT-PCR). The effects of LMCD1-AS1 knockdown on cell proliferation, migration and apoptosis were detected by colony formation assay, EdU assay, wound healing assay and TUNEL assay. Sphere formation assay was applied to assess sphere formation ability of THCA cells. Bioinformatics analysis and mechanism experiments, including ChIP assay, RIP assay and luciferase reporter assay were conducted to evaluate the downstream and upstream molecular mechanisms of LMCD-AS1.

Results: A marked up-regulation of LMCD1-AS1 in THCA cells relative to normal control cells was found. LMCD1-AS1 silencing suppressed proliferation and migration but induced apoptosis in THCA cells. Moreover, LMCD1-AS1 knockdown reduced the sphere formation capacity of THCA cells. The transcriptional factor GLI family zinc finger 2 (GLI2) binds to LMCD1-AS1, which contributed to LMCD1-AS1 up-regulation in THCA cells. Cytoplasmic LMCD1-AS1 sponged a shared microRNA between LMCD1-AS1 and GLI2. GLI2 was inhibited bymiR-1287-5p and disinhibited by LMCD1-AS1.

Conclusions: LMCD1-AS1exerts pro-tumorigenic function through sponging miR-1287-5p to elevate GLI2 expression in THCA development, constituting a feedback loop of LMCD1-AS1/miR-1287-5p/GLI2.

Keywords: LMCD1 antisense RNA 1 (LMCD1-AS1); miR-1287-5p; GLI family zinc finger 2 (GLI2); thyroid cancer (THCA)

Submitted Sep 14, 2020. Accepted for publication Nov 12, 2020.

doi: $10.21037 / \mathrm{atm}-20-7182$

View this article at: http://dx.doi.org/10.21037/atm-20-7182 


\section{Introduction}

Thyroid cancer (THCA) is the most commonly occurring endocrine malignancy, and contributes to a large proportion of endocrine-related mortalities $(1,2)$. The histotype of THCA ranges from well-differentiated THCA to poorly differentiated THCA and medullary THCA (3). The metastasis and invasion properties of THCA render the treatment process complex, along with the globally escalating incidence rate (4-6). Therefore, finding effective potential therapeutic targets for THCA management is vital.

Endogenous cellular RNA s over 200nt in length are long noncoding RNAs (lncRNAs), without an open reading frame of vital length $(7,8)$. Tumor inhibitor or tumor facilitator roles of lncRNA have been unveiled by recent studies, and have been implicated in various complex cellular processes $(9,10)$. For example, lncRNA-RP11-317J10.2 has been shown to promote proliferation and invasion in colorectal cancer (11). Furthermore, lncRNA APOC1P1-3 was shown to inhibit apoptosis by decreasing $\alpha$-tubulin acetylation in breast cancer (12). In lung cancer cells, lncRNA SNHG7 was demonstrated to accelerate proliferation, migration, and invasion, and suppressed apoptosis by up-regulating FAIM2 expression (13). Furthermore, lncRNAs as critical modulators of gene expression are arousing much interest from multiple research groups focusing on tumor development, in the context of competing endogenous RNA(ceRNA) (14). For example, the lncRNA AFAP1-AS1 has been shown to regulate the progression of pancreatic cancer via sequestration of miR-133a, thereby up-regulating the IGF1R oncogene (15). Furthermore, LINC01133 as a ceRNA inhibited gastric cancer progression through antagonizing the suppression of $\mathrm{miR}-106 \mathrm{a}-3 \mathrm{p}$ to regulate APC expression (16). LncRNA ILF3-AS1 as a ceRNA contributed to osteosarcoma progression via SOX5 upregulation by sponging miR-212 (17). LMCD1 antisense RNA 1 (LMCD1-AS1), has been demonstrated to facilitate tumor progression in cholangiocarcinoma (18) and hepatocellular carcinoma (HCC) (19). Whilst, the available research around the function of LMCD1-AS1 is limited, let alone a comprehensive understanding of its mechanisms in THCA.

Therefore, the present study aimed to unravel the biological function and modulatory mechanism of LMCD1AS1 in THCA.

We present the following article in accordance with the MDAR reporting checklist (available at http://dx.doi. org/10.21037/atm-20-7182).

\section{Methods}

\section{Cell culture}

The cell lines used in this study including THCA cells (KTC-3, TPC-1, BCPAP, K1) and normal thyroid epithelial cells (Nthy-ori3-1). Cells were commercially obtained from American Type Culture Collection (ATCC, Manassas, VA, USA). The medium used for all cell culture was RPMI1640 (Thermo Fisher Scientific, Waltham, MA, USA), supplemented with $10 \%$ fetal bovine serum (Hyclone, South Logan, UT, USA, $100 \mu \mathrm{g} / \mathrm{mL}$ streptomycin (Sigma-Aldrich, USA), and $100 \mathrm{U} / \mathrm{mL}$ penicillin. Cells were maintained in an incubator at $37{ }^{\circ} \mathrm{C}$ with $5 \% \mathrm{CO}_{2}$.

\section{Cell transfection}

The LMCD1-AS1 silencing plasmids (sh/LMCD1AS1\#1/2/3) and its negative control (sh/ctrl), GLI family zinc finger 2 (GLI2)-targeting vectors (sh/GLI2\#1/2), the GLI2 overexpression plasmid (pcDNA3.1/GLI2), and an empty vector were provided by GenePharma (Shanghai, China). RiboBio (Guangzhou,China) synthesized and provided miR-1287-5p mimics and inhibitor, and negative controls (NC mimics, NC inhibitor). Transfection of these plasmids was carried out using Lipofectamine ${ }^{\mathrm{TM}} 2000$ reagent (Thermo Fisher Scientific, USA).

\section{$R N A$ isolation and quantitative real-time polymerase chain reaction ( $q R T-P C R)$}

RNA was isolated from THCA cells using the TRIzol reagent (Invitrogen, CA, USA). Thunderbird SYBR qPCR Mix (Toyobo, Osaka, Japan) in an Applied Biosystems QuantStudio 5 RT-PCR System (Applied Biosystems, Foster City, CA, USA) was utilized for the qRT-PCR assays. For thermal cycling, samples were denatured for $30 \mathrm{~s}$ at $95{ }^{\circ} \mathrm{C}$, annealed for $45 \mathrm{~s}$ at $56^{\circ} \mathrm{C}$, and extended for $45 \mathrm{~s}$ at $72{ }^{\circ} \mathrm{C}$. Each PCR reaction was performed for 35 cycles. The relative expression of LMCD1-AS1, GLI2 and miR-1287$5 \mathrm{p}$ was calculated using the $2^{-\Delta \Delta \mathrm{Ct}}$ method, with GAPDH and U6 as the reference gene. Sequences for primers used in this study were listed in Table S1.

\section{Northern blot}

Northern blot was used for the measurement of LMCD1AS1 levels with an Ambion Northern Max-Gly Kit (Austin, TX, USA). A positively charged nylon membrane was 
utilized for total RNA electrophoresis and siphon, followed by UV cross-linking of RNA for the fixation of RNA to the membrane. Afterwards, pre-hybridization of crosslinked membranes with ULTRAhyb was performed, and an LMCD1-AS1-specific probe was adopted for RNA detection in roller bottles with digoxigenin-ddUTP (Roche Diagnostics, Indianapolis, IN, USA).

\section{Western blot}

Protein was isolated from THCA cells using RIPA buffer (Beyotime, Nanjing, China). Proteins were separated using 10\% SDS-PAGE and later transferred onto PVDF membrane (Millipore, Boston, USA). Membranes were blocked with $5 \%$ skim milk, then incubated with the primary antibodies anti-OCT4 (Cell Signaling Technology, Danvers, MA, USA), anti-NANOG (Cell Signaling Technology), anti-GLI2 (Abcam, Cambridge, UK), and anti- $\beta$ Actin (Proteintech, Wuhan, China) for 8 hours at $4{ }^{\circ} \mathrm{C}$. Horseradish peroxidase-conjugated anti-rabbit antibody (Cell Signaling Technology) was used as the secondary antibody. An enhanced chemiluminescence detection kit (Invitrogen) was used for blot detection.

\section{Subcellular fractionation}

A PARIS ${ }^{\text {TM }}$ Kit (Ambion, Austin, TX, USA) was used for subcellular fractionation to separate cytoplasmic and nuclear RNA according to the manufacturer's instructions. We collected and re-suspended $1 \times 10^{7}$ BCPAP and K1 cells in cell fraction buffer. Subsequently, 10 minutes' cultivation on ice was performed. Following centrifugation, the supernatant was discarded. Cell disruption buffer was then used for acquiring the nuclear pellet. The fractionation indicators GAPDH (cytoplasm), U6 (nucleus), and LMCD1-AS1 were assayed by qRT-PCR.

\section{Fluorescence in situ bybridization (FISH)}

An RNA FISH assay in BCPAP and K1 cells was performed using the Ribo ${ }^{\mathrm{TM}}$ Fluorescent in Situ Hybridization Kit (RiboBio Company, China) to determine LMCD1AS1 cellular distribution. The LMCD1-AS1 probe was synthesized by RiboBio, then labeled with Cy3 fluorescent dye. Probe sequence was listed in Table S2. The FISH assay was performed according to the manufacturer's instructions (RiboBio), followed by confocal laser-scanning microscopy (Leica, Germany).

\section{Dual-luciferase reporter assay}

The fragments of LMCD1-AS1 covering the binding sites of miR-1287-5p and the fragments of GLI2 covering the binding sites of miR-1287-5p were separately cloned into the dual reporter vector pmirGLO (Promega, Madison, WI, USA), then named LMCD1-AS1-WT or GLI2-WT. The mutated type of LMCD1-AS1 and GLI2 reporters were constructed by mutating miR-1287-5p binding sites, then named LMCD1-AS1-MUT and GLI2-MUT. Cotransfection of reporters with NC-mimics or miR-1287-5p mimics was carried out using Lipofectamine ${ }^{\mathrm{TM}} 2000$ (Invitrogen). A Dual-Luciferase Reporter System (Promega, Madison, USA) after a 48-hour incubation monitored the firefly or renilla luciferase activities (internal control).

\section{RNA immunoprecipitation (RIP)}

An EZ-Magna RIP Kit (Millipore, Bellerica, MA,USA) was used for RIP assays designed to probe the interplay between miR-1287-5p and LMCD1-AS1 or GLI2. Complete RIP lysis buffer was first used for lysing BCPAP and K1 cells, after which cell lysates went through cultivation at $4{ }^{\circ} \mathrm{C}$ with RIP buffer for 4 hours. Magnetic beads were precoated with anti-Ago2 to capture the RNA complex. AntiIgG was used as an internal control. The RNA complex was subsequently tested and validated by qRT-PCR.

\section{RNA pull-down}

A Biotin RNA Labeling Mix (Roche Diagnostics, Indianapolis, IN, USA) was utilized for the biotinylation of miR-1287-5p sense and miR-1287-5p antisense. Products were named bio-miR-1287-5p sense and bio-miR-1287-5p antisense. Following transfection of biotinylated products into BCPAP and K1cells for 48 hours, lysed BCPAP and K1 cells were treated with streptavidin-coated magnetic beads for 3 hours and incubated at $4{ }^{\circ} \mathrm{C}$. RNeasy Mini Kit (Qiagen) was then used for exploring bound RNAs, followed by qRT-PCR.

\section{Chromatin immunoprecipitation (ChIP)}

A Magna ChIP Kit (Millipore, Bedford, MA, USA) was used to analyze the relationship between the LMCD1AS1 promoter and GLI2 in a ChIP assay. As per the manufacturer's instructions, BCPAP and K1 cells were treated with formaldehyde to generate DNA-protein cross-links. Sonicated cell lysates were then produced for 
generating chromatin fragments. A GLI2 antibody was used to incubate the lysates for immunoprecipitation with $\mathrm{IgG}$ as the control. Following precipitated chromatin DNA restoration, qRT-PCR of DNA levels was conducted.

\section{Terminal-deoxynucleoitidyl Transferase Mediated Nick End Labeling (TUNEL) assay}

A TUNEL assay was performed in THCA cells to measure cell apoptosis using a cell death detection kit (Intergen Co., USA). After a 30-minute fixation with $4 \%$ paraformaldehyde at $25^{\circ} \mathrm{C}$, the cells were permeabilized for 20 minutes with proteinase $\mathrm{K}(20 \mu \mathrm{g} / \mathrm{mL})$ at room temperature. Slides were washed 3 times then underwent treatment with terminal deoxynucleotidyl transferase (TdT), Alexa Fluor 488-12dUTP Labeling Mix, and TdT Enzyme at $37^{\circ} \mathrm{C}$. DAPI was used for nuclear staining, and an Olympus DP72 fluorescence microscope (Olympus, Tokyo, Japan) was used for cell observation and imaging.

\section{Colony formation assay}

The clonogenic activities of BCPAP and K1 cells were analyzed using a colony forming assay. Briefly, transfected BCPAP and K1 cells were first prepared in single-cell suspensions, then maintained in a $2.5 \mathrm{~cm}$ well for 2 weeks. Paraformaldehyde combined with crystal violet was utilized for colony staining and visualization.

\section{Sphere formation assay}

Serum-free DMEM/F12 (Gibco, Carlsbad, CA, USA) supplemented with $20 \mathrm{ng} / \mathrm{mLEGF}$ (Sigma, St. Louis, MO, USA), 2\% (v/v) B27 (Invitrogen, Carlsbad, CA, USA), and $20 \mathrm{ng} / \mathrm{mLbasic}$ FGF (BD Biosciences, CA, USA) was utilized for sphere formation assays. Single BCPAP and K1 cells after dissociation were re-suspended in $2 \mathrm{~mL}$ medium, followed by cell propagation in an ultra-low attachment plate (6-well; Corning, NY, USA) under $5 \% \mathrm{CO}_{2}$ at $37{ }^{\circ} \mathrm{C}$. At day 10 or 14, we quantified the sphere numbers and diameters $(>50 \mu \mathrm{m})$ with an Olympus microscope.

\section{Wound bealing assay}

After plating and cultivating $3 \times 10^{5}$ cells in 6 -well plates, fully confluent cells were scratched in the wells using pipette tips. After culturing in serum-free medium for 24 hours, wound width were imaged and measured via microscopy (Olympus Corporation, Tokyo, Japan).

\section{5-Ethynyl-20-deoxyuridine (EdU) assay}

$\mathrm{K} 1$ and BCPAP cell proliferation was examined using the (5-Ethynyl-20-deoxyuridine) assay kit (KeyGen Biotech, Nanjing, Jiangsu, China) according to the manufacturer's instructions. Following transfection with the indicated vectors as described previously, cells were treated with EdU solution and incubated for 60-120 minutes. Nuclei of K1 and BCPAP cells were stained using DAPI. A BM-38XII microscope (BM instrument, Jiading, Shanghai, China) was used for fluorescence observation and imaging.

\section{Statistical analysis}

All experiments were repeated 3 times. Data were presented as mean \pm standard deviation. Difference between two groups was compared using student $t$ test, while difference among more than two groups was analyzed using oneway ANOVA. $\mathrm{P}$ value less than 0.05 indicated statistical significance. SPSS 20.0 software was used for all statistical analyses (Chicago, IL, USA).

\section{Results}

\section{Knockdown of LMCD1-AS1 inbibits malignant phenotypes of THCA cells}

We first probed LMCD1-AS1 expression in Nthy-ori3-1 (control), KTC-3, TPC-1, BCPAP, and K1cell lines. LMCD1-AS1 was exclusively up-regulated in KTC3, TPC-1, BCPAP, and K1 cells, but not in Nthy-ori3-1 (Figure 1A). Among the tumor cell lines, BCPAP and K1 had relatively higher expression of LMCD1-AS1, and thus were selected for subsequent experiments. LMCD1AS1 was depleted to evaluate its effect on BCPAP and K1 cell processes, and the knockdown efficacy was confirmed by qRT-PCR (Figure 1B). As presented by the results of colony formation assay and EdU assay, cell proliferation was impeded after LMCD1-AS1 silencing (Figure 1C,D and Figure S1A). Contrastingly, the TUNEL assay demonstrated a trend towards increased apoptosis in BCPAP and K1 cells following LMCD1-AS1 depletion (Figure $1 E$ and Figure S1B). Consistently, LMCD1-AS1 down-regulation also slowed migration of BCPAP and $\mathrm{K} 1$ cells in wound healing assays (Figure $1 F$ ). Meanwhile, 
A

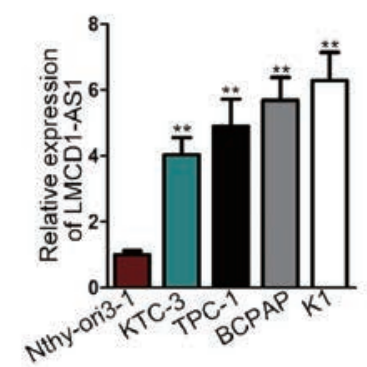

B

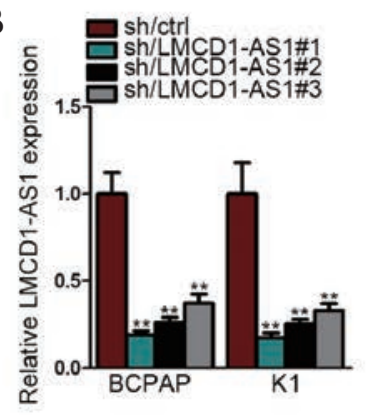

C
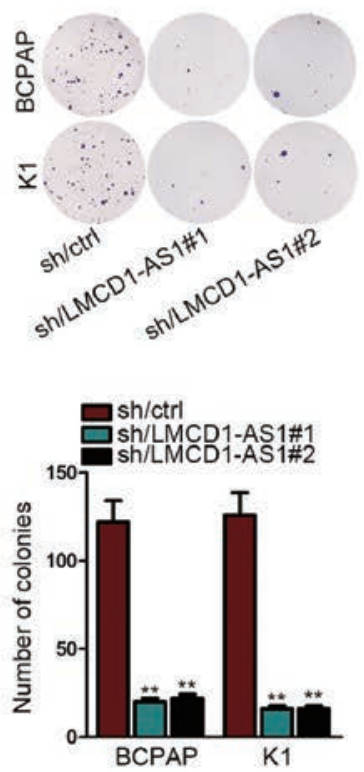

D

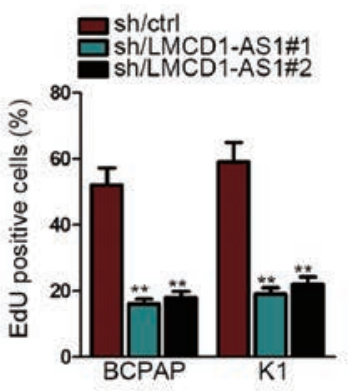

E

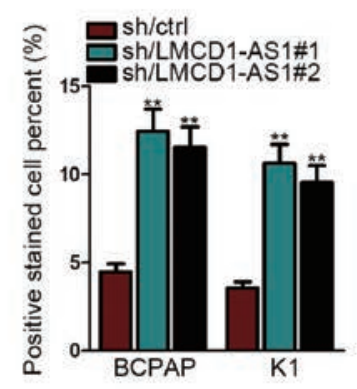

$\mathrm{F}$

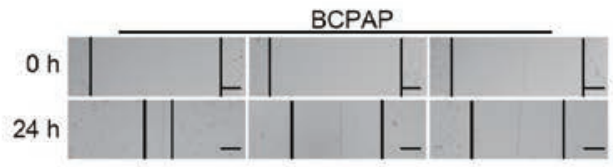

$\mathrm{K} 1$
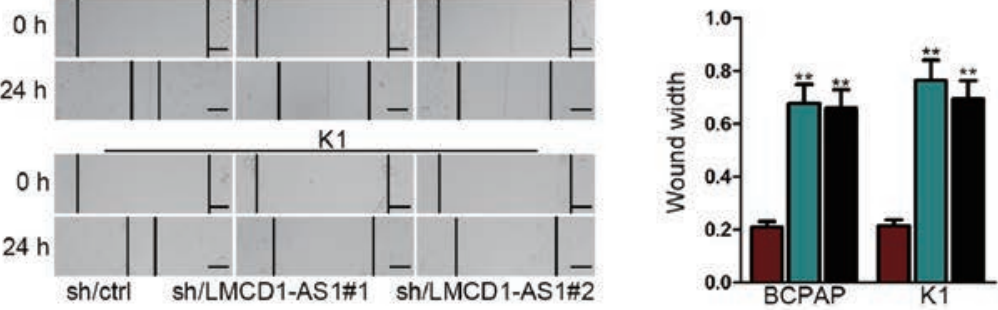

sh/ctrl

sh/LMCD1-AS1\#1
sh/LMCD1-AS1\#2

G

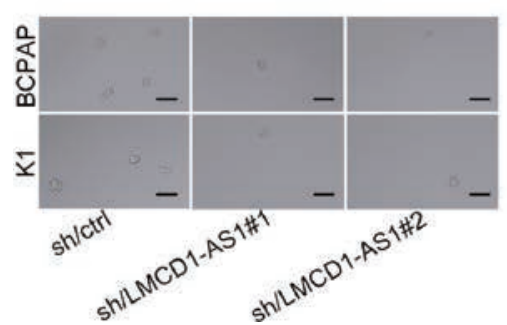

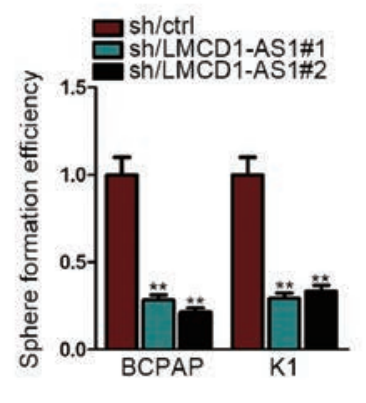

$\mathrm{H}$

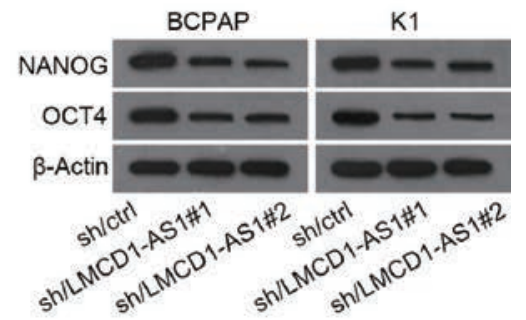

Figure 1 Knockdown of LMCD1-AS1 inhibits malignant phenotypes of THCA cells. (A) qRT-PCR analysis was conducted in KTC-3, TPC-1, BCPAP, K1 and Nthy-ori3-1 (control) cells to examine LMCD1-AS1 expression. (B) LMCD1-AS1 expression was measured in response to sh-LMCD1-AS1\#1/2/3 or sh-NC. (C,D) BCPAP and K1 cell proliferation was detected using colony formation assay (colonies were visualized by crystal violet staining) and EdU assay following transfection with sh-LMCD1-AS1\#1/2/3 or sh-NC. (E) BCPAP and K1 cell apoptosis was measured via TUNEL assay. (F) LMCD1-AS1-downregulated BCPAP and K1 cells were monitored in wound healing assays (scale bar: $200 \mu \mathrm{m}$ ). (G) Sphere formations of BCPAP and K1 cells following LMCD1-AS1 knockdown were examined (scale bar: $200 \mu \mathrm{m})$. (H) Western blot assay of the stemness proteins (NANOG and OCT4). **, $\mathrm{P}<0.01$. LMCD1-AS1, LMCD1 antisense RNA 1; THCA, thyroid cancer; qRT-PCR, quantitative real-time polymerase chain reaction; EdU, 5-Ethynyl-20-deoxyuridine; TUNEL, Terminaldeoxynucleoitidyl Transferase Mediated Nick End Labeling. 
accompanying the reduced LMCD1-AS1 level, stem-like activity of BCPAP and $\mathrm{K} 1$ cells was impaired, highlighted by a decline in sphere number (Figure 1G). Furthermore, the mRNA and protein levels of stemness-associated markers (OCT4 and NANOG) were all decreased following LMCD1-AS1 depletion (Figure $1 H$ and Figure S1C). Taken together, these results suggested that LMCD1AS1 is overexpressed in THCA cells, and LMCD1-AS1 knockdown suppresses the malignant phenotypes of THCA cells.

\section{GLI2 is a transcriptional activator of LMCD1-AS1}

Following a search using the JASPAR database (http:// jaspar.genereg.net/), we uncovered that within the LMCD1AS1 promoter, there were 2 potential GLI2-binding sites (Figure 2A). Therefore, we speculated that GLI2 might facilitate LMCD1-AS1 expression through their specific binding. Western blot and qRT-PCR analyses confirmed the efficacy of GLI2 knockdown in BCPAP and K1 cells (Figure 2B). GLI2 was increased after pcDNA3.1/GLI2 transfection (Figure 2C). We then estimated the expression level of LMCD1-AS1 following GLI2 alteration in BCPAP and K1 cells. Northern blot and qRT-PCR demonstrated that GLI2 positively modulated LMCD1-AS1 expression (Figure 2D,E). Luciferase reporter assays indicated that GLI2 had an affinity to both site 1 and site 2 segments in the LMCD1-AS1 promoter. However, activities of the LMCD1-AS1 promoter remained intact after mutations at both sites (Figure 2F,G). The ChIP assay further confirmed the results that GLI2 binds to the LMCD1-AS1 promoter (Figure 2H). Overall, these results indicated that LMCD1AS1 transcriptionally activates GLI2.

\section{LMCD1-AS1 sequesters miR-1287-5p to facilitate GLI2 expression}

It is known that the cellular distribution of lncRNAs directly impact their regulatory mechanism. Hence, the presence of LMCD1-AS1 was interrogated in BCPAP and K1 cells. Cell fractionation demonstrated LMCD1-AS1 was abundant in the cytoplasm, also confirmed by FISH assay (Figure $3 A$ and Figure S1D). Given the cytoplasmic presence of LMCD1AS1 in cells, we adopted a well-characterized ceRNA network to locate the downstream miRNA of LMCD1AS1. As demonstrated in Figure 3B, 11 shared miRNAs of LMCD1-AS1 were found. Thus, the identification of target
miRNAs was conducted. It demonstrated that LMCD1AS1 activity declined in the presence of miR-1287-5p, miR4500, let-7c-5p or miR-98-5p overexpression (Figure 3C). Nevertheless, only miR-1287-5p was accelerated following LMCD1-AS1 depletion (Figure 3D). The predicted binding sites between LMCD1-AS1 and miR-1287-5p were then found. Luciferase activity of LMCD1-AS1-WT in cells transfected with miR-1287-5p mimics was reduced (Figure 3E). LMCD1-AS1 precipitated by bio-miR-1287$5 \mathrm{p}$ sense increased compared to that in bio-miR-1287$5 \mathrm{p}$ antisense (Figure $3 F$ ). Similarly, the luciferase activity of GLI2-WT declined in the presence of miR-1287$5 \mathrm{p}$ mimics, while no significant change was found in the GLI2-MUT group (Figure $3 G$ ). In addition, the binding of miR-1287-5p to GLI2 was re-validated in an RNA pulldown assay (Figure $3 H$ ). RIP confirmed the co-expression of LMCD1-AS1, miR-1287-5p, and GLI2 in the antiAgo2 group, implying their presence in the RNA-induced silencing complex (Figure 3I). Additional qRT-PCR demonstrated that GLI2 was restrained by miR-1287-5p upregulation (Figure 37). Furthermore, the suppressed GLI2 mRNA and protein induced by LMCD1-AS1 depletion were recovered by miR-1287-5p inhibition (Figure $3 \mathrm{~K}$ ). Taken together, LMCD1-AS1 acts as a miR-1287-5p sponge to boost GLI2 expression in THCA cells.

\section{MiR-1287-5p and GLI2 rescue THCA cells from the impact of LMCD1-AS1}

We further verified that the carcinogenic function of LMCD1-AS1 was dependent on GLI2, a target of miR-1287-5p. Colony formation assay and EdU assay demonstrated that the slowed cell proliferation induced by LMCD1-AS1 was reversed after GLI2 overexpression (Figure $4 A, B$ and Figure S1E). Overexpression of GLI2 counteracted LMCD1-AS1 depletion-mediated apoptosis in TUNEL assays (Figure $4 C$ and Figure S1F). Attenuated cell migration after LMCD1-AS1 depletion was recovered through GLI2 alteration (Figure 4D). Impaired sphere formation capacity induced by LMCD1AS1 was potentiated with the restored expression of GLI2 (Figure 4E). Moreover, sh/LMCD1-AS1\#1-induced suppression on OCT4 and NANOG expression was abolished after GLI2 overexpression (Figure $4 F$ and Figure S1G). Altogether, overexpression of GLI2 reverses the inhibitory effect of LMCD1-AS1 depletion on THCA cell proliferation, migration, and stemness. 
A

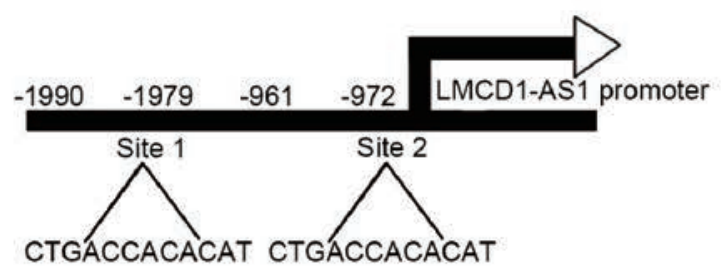

C

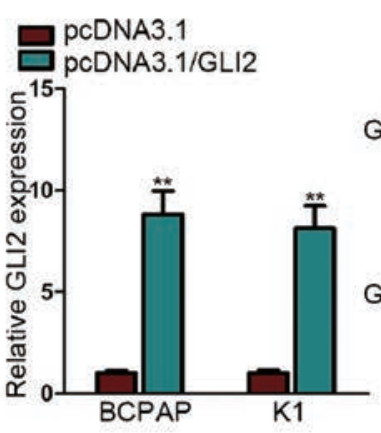

B

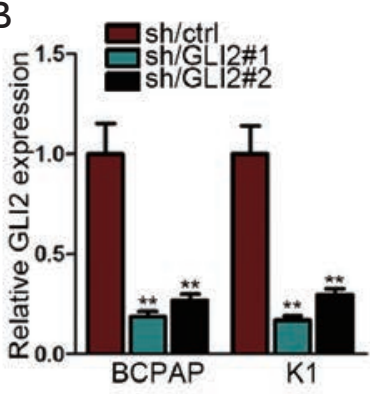

$\mathrm{D}$

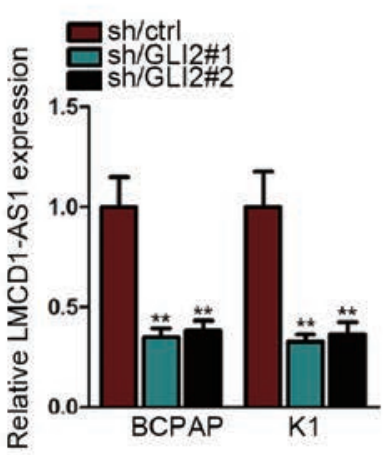

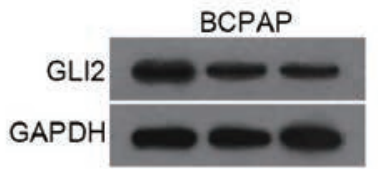

K1
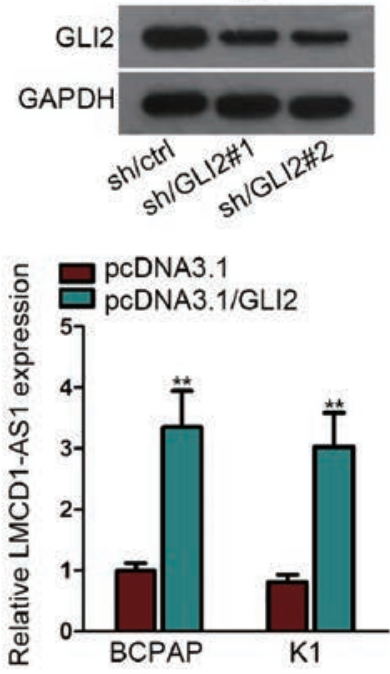

$E$

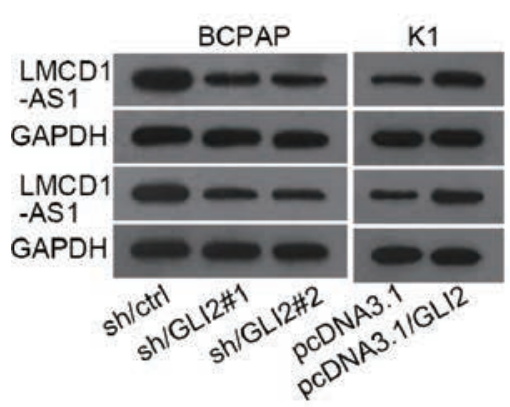

G

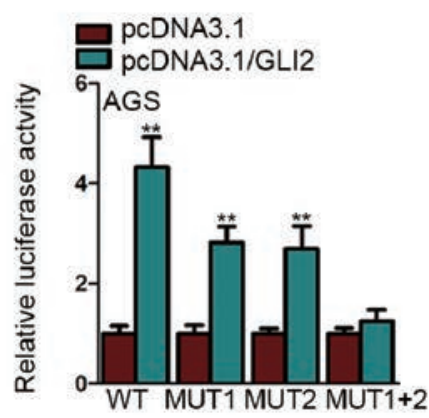

$\mathrm{F}$
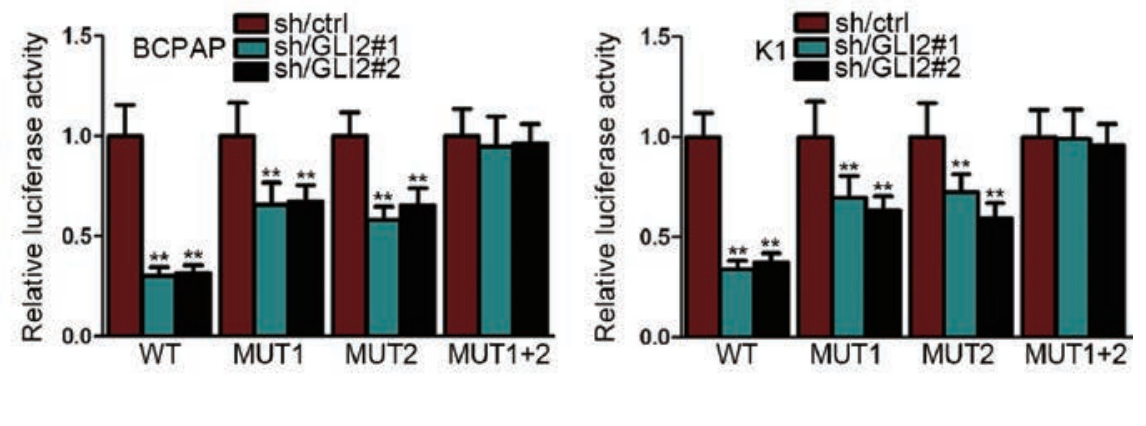
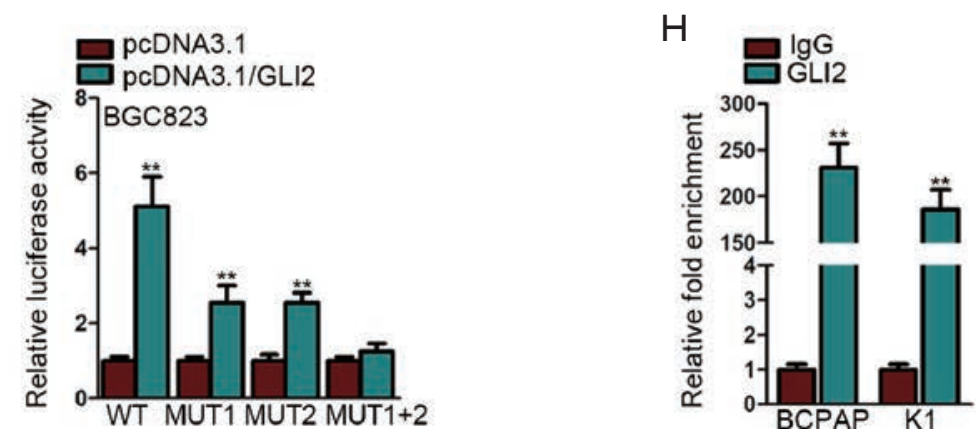

Figure 2 GLI2 is a transcriptional activator of LMCD1-AS1. (A) Analysis of GLI2 binding sites in the LMCD1-AS1 promoter. (B,C) GLI2 mRNA and protein in BCPAP and K1 cells were measured using qRT-PCR and western blot following GLI2 silencing and overexpression. (D,E) qRT-PCR and northern blot analysis of LMCD1-AS1 expression level following GLI2 alteration. (F,G) The effects of GLI2 on LMCD1-AS1 promoter activity were determined using luciferase reporter assays. $(\mathrm{H})$ The interplay between GLI2 and the LMCD1-AS1 promoter was explored using ChIP assay. ${ }^{* *}, \mathrm{P}<0.01$. GLI2, GLI family zinc finger 2; LMCD1-AS1, LMCD1 antisense RNA 1; qRT-PCR, quantitative real-time polymerase chain reaction; ChIP, chromatin immunoprecipitation. 


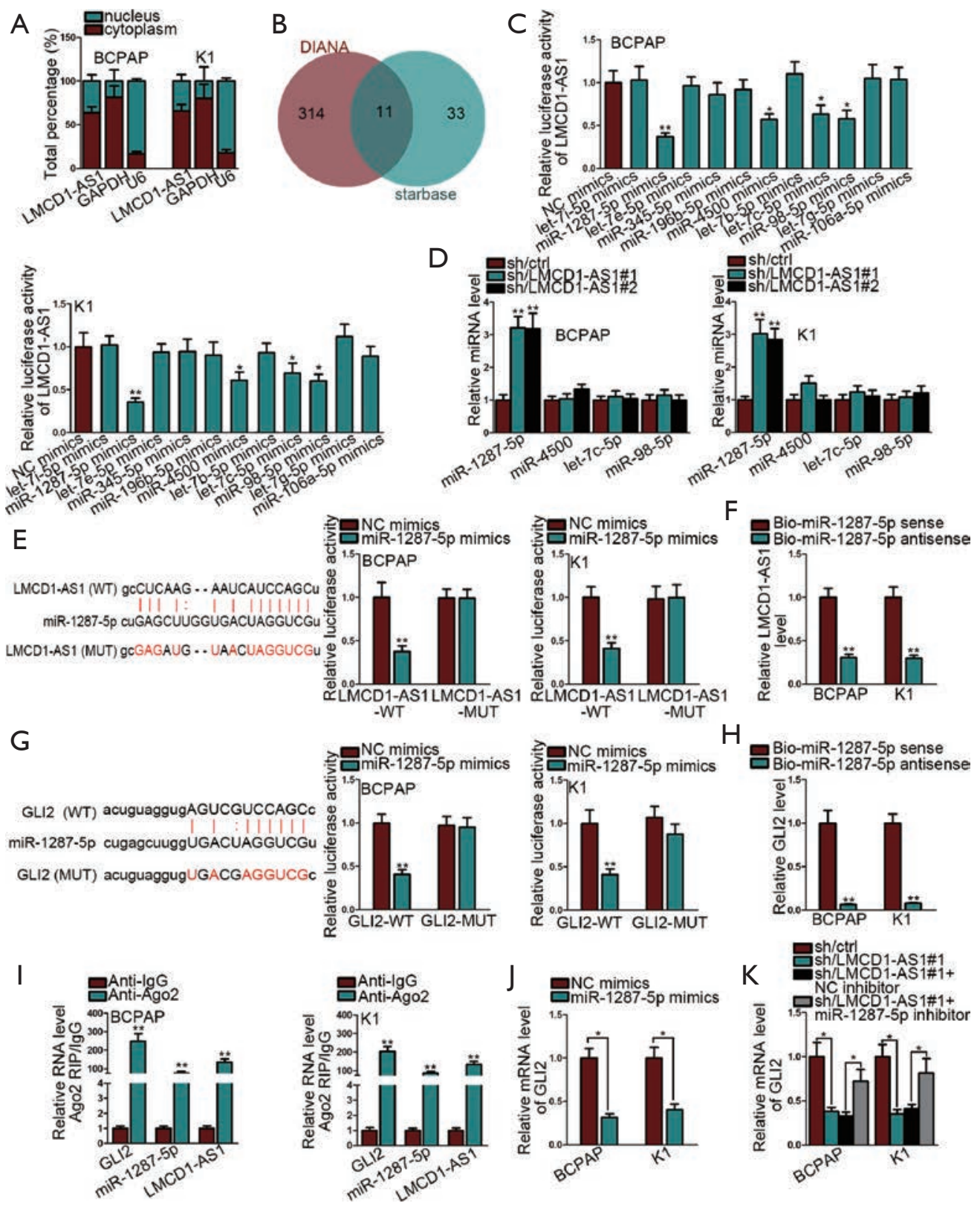

Figure 3 LMCD1-AS1 sequesters miR-1287-5p to facilitate GLI2 expression. (A) The cellular distribution of LMCD1-AS1 was verified by subcellular fractionation assay. (B) Shared miRNAs of LMCD1-AS1 from the starBase and DIANA databases were found. (C) Luciferase reporter assay revealed LMCD1-AS1 activity declined upon overexpression of the 11 shared miRNAs. (D) Expression changes of miR1287-5p, miR-4500, let-7c-5p, and miR-98-5p in the absence of LMCD1-AS1 determined using qRT-PCR. (E) The interaction between LMCD1-AS1 and miR-1287-5p was measured in luciferase reporter experiments. (F) The interaction between LMCD1-AS1 and miR-1287$5 p$ was measured by RNA pull-down assays. (G) The combination of GLI2 WT or mutation and miR-1287-5p was evaluated by luciferase reporter activity assay. (H) RNA pull-down assay detected the interplay between LMCD1-AS1 and miR-1287-5p. (I) Ago2-RIP assay was used to monitor the endogenous RNAs (GLI2, miR-1287-5p and LMCD1-AS1) associated with Ago2; IgG was used as the negative control. RNA enrichment was evaluated by qRT-PCR. (J) GLI2 mRNA was assessed after transfection with NC mimics or miR-1287-5p mimics. (K) The decreased mRNA level of GLI2 in response to LMCD1-AS1 depletion was partially abrogated after miR-1287-5p down-regulation. *, $\mathrm{P}<0.05,{ }^{* *}, \mathrm{P}<0.01$. LMCD1-AS1, LMCD1 antisense RNA 1; GLI2, GLI family zinc finger 2; qRT-PCR, quantitative real-time polymerase chain reaction; RIP, RNA immunoprecipitation. 

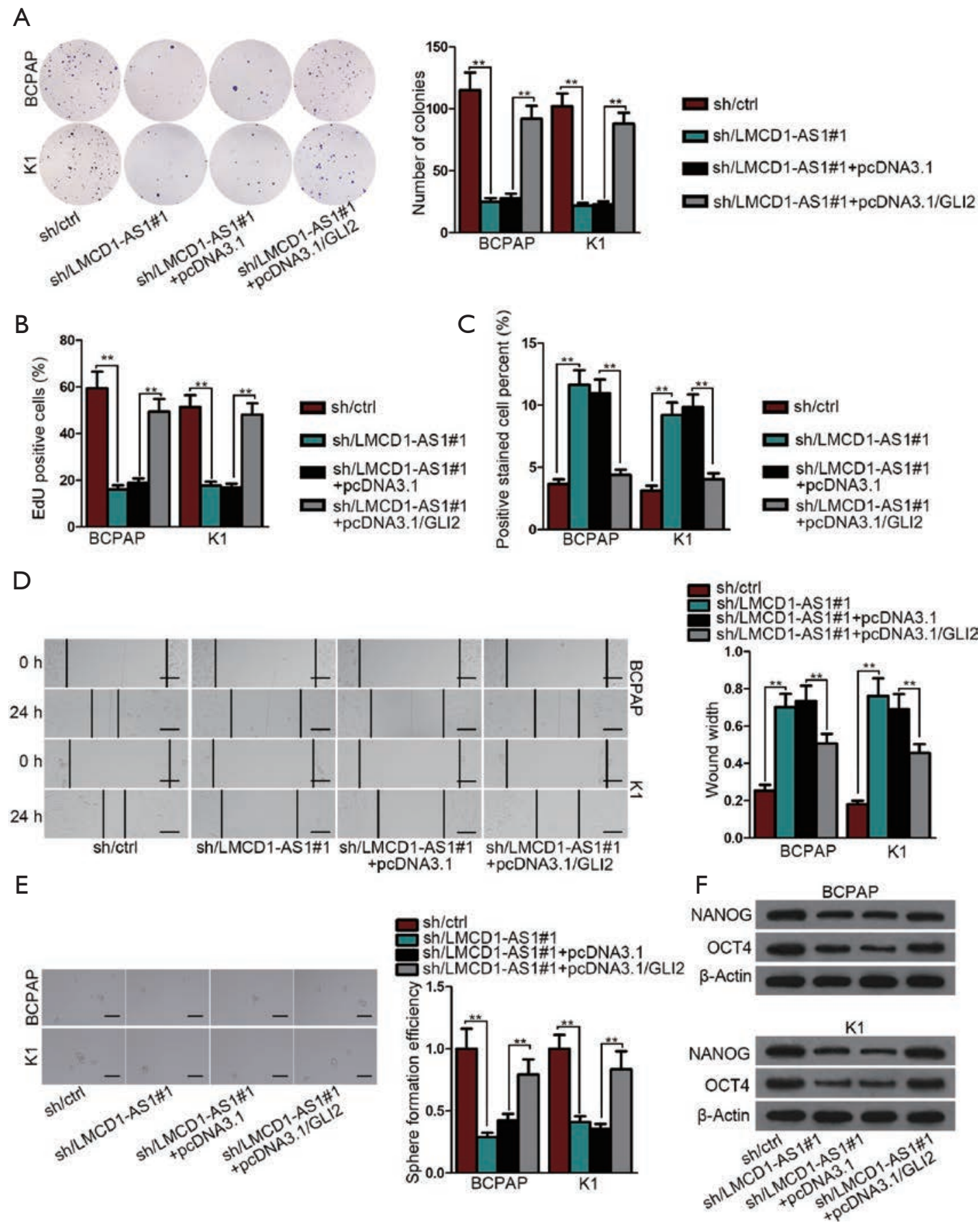

Figure 4 MiR-1287-5p and GLI2 rescue THCA cells from the impact of LMCD1-AS1. (A,B) The influence of pcDNA3.1/GLI2 on the proliferation of cells with LMCD1-AS1 knockdown was investigated using colony formation assay (colonies were visualized by crystal violet staining) and EdU assay. (C) TUNEL experiments probed the effects of pcDNA3.1/GLI2 on LMCD1-AS1 silencing-regulated apoptosis. (D) Wound healing assays probed the impact of GLI2 overexpression on LMCD1-AS1-depleted cell migration (scale bar: $200 \mu \mathrm{m})$. (E) Sphere formation capacity after GLI2 overexpression in LMCD1-AS1-inhibited BCPAP and K1 cells was restored (scale bar: $200 \mu \mathrm{m})$. (F) Western blot measured the protein levels of stemness markers in LMCD1-AS1-depleted cells following the transfection of pcDNA3.1/GLI2 plasmids. **, P<0.01. GLI2, GLI family zinc finger 2; THCA, thyroid cancer; LMCD1-AS1, LMCD1 antisense RNA 1; EdU, 5-Ethynyl20-deoxyuridine; TUNEL, Terminal-deoxynucleoitidyl Transferase Mediated Nick End Labeling. 


\section{Discussion}

Mounting evidence is highlighting THCA as one of the most common endocrine malignancies, and its invasion and metastasis properties contribute to the high incidence of endocrine-related deaths $(2,4)$. Despite the broad investigations into lncRNA dysregulation in THCA, the underlying lncRNA-modulated mechanisms remain to be understood.

Recently, IncRNAs have been demonstrated to be tumor inhibitors or promotors in various types of cancers, and have implicated certain gene expression and multiple malignant cellular phenotypes. For example, the lncRNA MALAT1 facilitates proliferation, invasion, and migration via SOX9 regulation in colorectal cancer (20). Furthermore, lncRNA NORAD augments SIP1 expression to accelerate cell proliferation and invasion in cervical cancer (21). LncRNA LOC441178 decreases cell invasion and migration in squamous carcinoma by targeting ROCK1 (22). LMCD1AS1 is an oncogene, which has been shown to promote tumor progression in HCC and cholangiocarcinoma. However, its overexpression in THCA cells is uncovered in this study for the first time. In this study, we determined the upregulation of LMCD1-AS1 in THCA cells. Consistent with previous studies, LMCD1-AS1 promoted cell proliferation and migration and maintains stemness in THCA.

Mechanistically, lncRNAs can be upregulated by their upstream transcription activators $(23,24)$. Here, we revealed that in THCA cells, LMCD1-AS1 was transcriptionally boosted by transcription activator GLI2. As a core protein in Hedgehog $(\mathrm{Hh})$ signaling during tumorigenesis, GLI2 has been shown to be pro-tumorigenic in pancreatic cancer (25), bladder cancer (26), and ovarian cancer (27) amongst others. Additionally, GLI2 was tumor-promoting via FOXE1 in papillary thyroid carcinoma through the $\mathrm{Wnt} / \beta$-catenin pathway (28). In this study, we found that oncogenic GLI2 was a transcription activator of LMCD1AS1, supporting its role in THCA regulation. Importantly, lncRNAs can regulate their transcriptional regulators in turn and thus form a positive feedback loop. For instance, SP1-DLEU1-miR-4429 positive feedback loop facilitates cell growth in glioblastoma $(29,30)$. LncRNA HEGBC promotes gallbladder cancer progression by forming a positive feedback loop with IL11/STAT3 signaling pathway (31). CASC9/miR-519d/STAT3 positive feedback loop contributes to glioma tumor growth (32). In the current study, we uncovered that LMCD1-AS1 positively modulated its transcription activator GLI2 in THCA cells.

As for the mechanisms mediated by cytoplasmic lncRNAs, one notable mechanism is the ceRNA network, in which IncRNA liberates target genes from the inhibition of miRNAs via competitively binding to miRNAs (14). Some well-recognized lncRNAs include AFAP1-AS1 (15), LINC01133 (16), and lncRNA ILF3-AS1 (17). LMCD1AS1 is also a well-described ceRNA for let-7g (19) and miR-345-5p (18). In this study, we revealed that LMCD1AS1 upregulated GLI2 through sponging miR-1287-5p in THCA cells, which formed a positive feedback loop.

This study, to the best of our knowledge, is the first study to reveal that LMCD1-AS1 sponges miR-1287-5p to enhance GLI2 expression in THCA. However, lack of in vivo data is a limitation of our current study. Thus, we will explore the effect of the positive feedback loop on in vivo tumor growth in our future study. Based on previous studies, lncRNAs can involve in multiple biological processes in human cancers through regulating downstream signaling pathways (33-36). GLI2 is a downstream transcription factor of hedgehog signaling pathway. Therefore, we will investigate whether LMCD1-AS1 exerted functions through activating hedgehog pathway in the next study.

\section{Conclusions}

Overall, LMCD1-AS1 acts as a molecular sponge of miR1287-5p to facilitate GLI2 expression, thereby contributing to THCA progression. GLI2 can transcriptionally facilitate LMCD1-AS1 expression. Therefore, a closed positive feedback loop is established by LMCD1-AS1, miR$1287-5 \mathrm{p}$, and GLI2 in THCA cell growth and stemness, enhancing the evidence for IncRNA-induced development and progression of THCA.

\section{Acknowledgments}

Funding: This study was supported by Major Special Projects of the Ministry of Science and Technology, a new strategy for accurate diagnosis and treatment of hepatitis B-related hepatocellular carcinoma with anti-recurrence and metastasis after operation [2017] (2017ZX10203207); National Natural Science Foundation of China, discussion on the mechanism of osteopontin interfering cholesterol metabolism and affecting cholesterol stone formation in gallbladder [2013] (81270536); National Natural Science Foundation of China, the role of osteopontin in the genesis of gallstones and its clinical application [2009] (30801105). 


\section{Footnote}

Reporting Checklist: The authors have completed the MDAR reporting checklist. Available at http://dx.doi.org/10.21037/ atm-20-7182

Data Sharing Statement: Available at http://dx.doi. org/10.21037/atm-20-7182

Conflicts of Interest: All authors have completed the ICMJE uniform disclosure form (available at http://dx.doi. org/10.21037/atm-20-7182). The authors have no conflicts of interest to declare.

Ethical Statement: The authors are accountable for all aspects of the work in ensuring that questions related to the accuracy or integrity of any part of the work are appropriately investigated and resolved.

Open Access Statement: This is an Open Access article distributed in accordance with the Creative Commons Attribution-NonCommercial-NoDerivs 4.0 International License (CC BY-NC-ND 4.0), which permits the noncommercial replication and distribution of the article with the strict proviso that no changes or edits are made and the original work is properly cited (including links to both the formal publication through the relevant DOI and the license). See: https://creativecommons.org/licenses/by-nc-nd/4.0/.

\section{References}

1. Blomberg M, Feldt-Rasmussen U, Andersen KK, et al. Thyroid cancer in Denmark 1943-2008, before and after iodine supplementation. Int J Cancer 2012;131:2360-6.

2. Xing M. Molecular pathogenesis and mechanisms of thyroid cancer. Nat Rev Cancer 2013;13:184-99.

3. Carling T, Udelsman R. Thyroid Cancer. Annual Review of Medicine 2014;65:125-37.

4. Wang Y, Guan Q, Xiang J, et al. Clinicopathologic features and prognostic factors of diffuse sclerosing variant of papillary thyroid carcinoma: a population-based analysis. Transl Cancer Res 2018;7:695-705.

5. Schonfeld SJ, Ron E, Kitahara CM, et al. Hormonal and reproductive factors and risk of postmenopausal thyroid cancer in the NIH-AARP Diet and Health Study. Cancer Epidemiol 2011;35:e85-90.

6. Liu C, Liu Y, Zhang L, et al. Risk factors for highvolume lymph node metastases in $\mathrm{cN} 0$ papillary thyroid microcarcinoma. Gland Surg 2019;8:550-6.

7. Wu Q, Li T, Zhu D, et al. Altered expression of long noncoding RNAs in peripheral blood mononuclear cells in patients with impaired leptomeningeal collaterals after acute anterior large vessel occlusions. Ann Transl Med 2019;7:523.

8. Wilusz JE, Sunwoo H, Spector DL. Long noncoding RNAs: functional surprises from the RNA world. Genes Dev 2009;23:1494-504.

9. Kung JT, Colognori D, Lee JT. Long noncoding RNAs: past, present, and future. Genetics 2013;193:651-69.

10. Wang KC, Chang HY. Molecular Mechanisms of Long Noncoding RNAs. Molecular Cell 2011;43:904-14.

11. Hu J, Tian J, Zhu S, et al. Sox 5 contributes to prostate cancer metastasis and is a master regulator of TGFbeta-induced epithelial mesenchymal transition through controlling Twist1 expression. Br J Cancer 2018;118:88-97.

12. Liao XH, Wang JG, Li LY, et al. Long intergenic noncoding RNA APOC1P1-3 inhibits apoptosis by decreasing alpha-tubulin acetylation in breast cancer. Cell Death Dis 2016; 7:e2236.

13. She K, Huang J, Zhou H, et al. lncRNA-SNHG7 promotes the proliferation, migration and invasion and inhibits apoptosis of lung cancer cells by enhancing the FAIM2 expression. Oncol Rep 2016;36:2673-80.

14. Qi X, Zhang DH, Wu N, et al. ceRNA in cancer: possible functions and clinical implications. J Med Genet 2015;52:710-8.

15. Chen B, Li Q, Zhou Y, et al. The long coding RNA AFAP1-AS1 promotes tumor cell growth and invasion in pancreatic cancer through upregulating the IGF1R oncogene via sequestration of miR-133a. Cell Cycle 2018;17:1949-66.

16. Yang XZ, Cheng TT, He QJ, et al. LINC01133 as ceRNA inhibits gastric cancer progression by sponging miR-106a$3 p$ to regulate APC expression and the $\mathrm{Wnt} /$ beta-catenin pathway. Mol Cancer 2018;17:126.

17. Hu XH, Dai J, Shang HL, et al. SP1-mediated upregulation of lncRNA ILF3-AS1 functions a ceRNA for miR-212 to contribute to osteosarcoma progression via modulation of SOX5. Biochem Biophys Res Commun 2019;511:510-7.

18. Yu J, Zhang B, Zhang H, et al. E2F1-induced upregulation of long non-coding RNA LMCD1-AS1 facilitates cholangiocarcinoma cell progression by regulating miR-345-5p/COL6A3 pathway. Biochem Biophys Res Commun 2019;512:150-5.

19. Wang T, Fu X, Jin T, et al. Aspirin targets P4HA2 through 
inhibiting NF-kappaB and LMCD1-AS1/let-7g to inhibit tumour growth and collagen deposition in hepatocellular carcinoma. EBioMedicine 2019;45:168-80.

20. Xu Y, Zhang X, Hu X, et al. The effects of lncRNA MALAT1 on proliferation, invasion and migration in colorectal cancer through regulating SOX9. Mol Med 2018;24:52.

21. Huo H, Tian J, Wang R, et al. Long non-coding RNA NORAD upregulate SIP1 expression to promote cell proliferation and invasion in cervical cancer. Biomed Pharmacother 2018;106:1454-60.

22. Xu K, Tian H, Zhao S, et al. Long Noncoding RNA LOC441178 Reduces the Invasion and Migration of Squamous Carcinoma Cells by Targeting ROCK1. Biomed Res Int 2018;2018:4357647.

23. Wang H, Huo X, Yang XR, et al. STAT3-mediated upregulation of lncRNA HOXD-AS1 as a ceRNA facilitates liver cancer metastasis by regulating SOX4. Mol Cancer 2017;16:136.

24. Liu HT, Liu S, Liu L, et al. EGR1-Mediated Transcription of lncRNA-HNF1A-AS1 Promotes CellCycle Progression in Gastric Cancer. Cancer Res 2018;78:5877-90.

25. Adams CR, Htwe HH, Marsh T, et al. Transcriptional control of subtype switching ensures adaptation and growth of pancreatic cancer. Elife 2019;8:e45313.

26. Raven PA, Lysakowski S, Tan Z, et al. Inhibition of GLI2 with antisense-oligonucleotides: A potential therapy for the treatment of bladder cancer. J Cell Physiol 2019;234:20634-47.

27. Zhang H, Wang Y, Chen T, et al. Aberrant Activation Of Hedgehog Signalling Promotes Cell Migration And Invasion Via Matrix Metalloproteinase-7 In Ovarian Cancer Cells. J Cancer 2019;10:990-1003.

28. Ma J, Huang X, Li Z, et al. FOXE1 supports the tumor

Cite this article as: Shao $\mathrm{J}, \mathrm{Xu} \mathrm{Y,} \mathrm{Li} \mathrm{H,} \mathrm{Chen} \mathrm{L,} \mathrm{Wang} \mathrm{W,}$ Shen D, Chen J. LMCD1 antisense RNA 1 (LMCD1-AS1) potentiates thyroid cancer cell growth and stemness via a positive feedback loop of miR-1287-5p/GLI2. Ann Transl Med 2020;8(22):1508. doi: 10.21037/atm-20-7182 promotion of Gli2 on papillary thyroid carcinoma by the Wnt/beta-catenin pathway. J Cell Physiol 2019;234:17739-48.

29. Liu X, Chen R, Liu L. SP1-DLEU1-miR-4429 feedback loop promotes cell proliferative and anti-apoptotic abilities in human glioblastoma. Biosci Rep 2019;39.

30. Wang Y, Cao R, Yang W, et al. SP1-SYNE1-AS1-miR525-5p feedback loop regulates Ang-II-induced cardiac hypertrophy. J Cell Physiol 2019;234:14319-29.

31. Yang L, Gao Q, Wu X, et al. Long noncoding RNA HEGBC promotes tumorigenesis and metastasis of gallbladder cancer via forming a positive feedback loop with IL-11/STAT3 signaling pathway. J Exp Clin Cancer Res 2018;37:186.

32. Liu H, Li C, Yang J, et al. Long noncoding RNA CASC9/ miR-519d/STAT3 positive feedback loop facilitate the glioma tumourigenesis. J Cell Mol Med 2018;22:6338-44.

33. Peng WX, Huang JG, Yang L, et al. Linc-RoR promotes MAPK/ERK signaling and confers estrogen-independent growth of breast cancer. Mol Cancer 2017;16:161.

34. Jiang L, Zhao XH, Mao YL, et al. Long non-coding RNA RP11-468E2.5 curtails colorectal cancer cell proliferation and stimulates apoptosis via the JAK/STAT signaling pathway by targeting STAT5 and STAT6. J Exp Clin Cancer Res 2019;38:465.

35. Huang Y, Zhang J, Hou L, et al. LncRNA AK023391 promotes tumorigenesis and invasion of gastric cancer through activation of the PI3K/Akt signaling pathway. J Exp Clin Cancer Res 2017;36:194.

36. Shang Z, Yu J, Sun L, et al. LncRNA PCAT1 activates $\mathrm{AKT}$ and $\mathrm{NF}-\kappa \mathrm{B}$ signaling in castration-resistant prostate cancer by regulating the PHLPP/FKBP51/IKK $\alpha$ complex. Nucleic Acids Res 2019;47:4211-25.

(English Language Editor: C. Betlazar-Maseh) 